\title{
Proyecto África «La Leyenda de Faro»: Efectos de una metodología basada en la gamificación sobre la motivación situacional respecto al contenido de expresión corporal en Educación Secundaria Africa Project «La Leyenda de Faro»: Effects of a methodology based on gamification on situational motivation about the content of Corporal Expression in Secondary Education \\ *Mateo Real Pérez, **David Sánchez Oliva, *Carmen Padilla Moledo \\ *Universidad de Cádiz (España), **Universidad de Extremadura (España)
}

Resumen. El objetivo del siguiente estudio fue contrastar el efecto de una intervención en una unidad didáctica (UD) aplicando metodologías activas como la gamificación, en comparación con otra en la que se utilizaron estilos de enseñanza tradicionales, sobre la motivación situacional respecto a los contenidos de expresión corporal, en alumnos de $3^{\circ}$ y $4^{\circ}$ de Educación Secundaria Obligatoria (ESO). Para ello, utilizamos la Danza Africana como eje vertebrador en las clases en Educación Física (EF), y se analizaron comparativamente diferentes variables motivacionales teniendo en cuenta la Teoría de la Autodeterminación (TAD) en 98 alumnos $(\mathrm{M}$ edad=15.5; DT=.537) divididos en un grupo control (n=49) y un grupo experimental $(\mathrm{n}=49)$. Las mediciones se llevaron a cabo antes y después del período de intervención a lo largo de la UD a través de cuestionarios que medían el apoyo a las necesidades psicológicas básicas (CANPB), la satisfacción de las NPB (BPNES), la motivación (CMEF) y el clima motivacional (PEPS; SSI-EF) para comprobar la eficacia de la intervención. Los resultados sugieren que utilizar metodologías activas innovadoras parece mostrarse como una herramienta eficaz sobre distintas variables motivacionales del alumnado como: apoyo a la autonomía, apoyo a las relaciones sociales, autonomía, motivación intrínseca, motivación identificada y motivación externa. A pesar de ello, son necesarios más estudios para determinar significativamente la influencia de la gamificación sobre la motivación en las clases de EF.

Palabras Clave: gamificación, expresión corporal, educación física, motivación, danza.

Abstract. The objective of the following study was to contrast the effect of an intervention in a didactic unit (DU) applying active methodologies such as gamification, in comparison with another in which traditional teaching styles were used, on situational motivation regarding the corporal expression contents, in Secondary Education students. For this, we used African Dance as a reference in Physical Education (PE) classes, and were comparatively analysed different motivational variables taking into account the Self-Determination Theory $(\mathrm{TAD})$ in 98 students $(\mathrm{M}$ age $=15.5$; $\mathrm{SD}=.537)$ divided into a control group $(n=49)$ and an experimental group $(n=49)$. Measurements were carried out before and after the intervention period throughout the DU through questionnaires that measured support for basic psychological needs (CANPB), satisfaction of NPBs (BPNES), motivation (CMEF) and motivational climate (PEPS; SSI-EF) to check the effectiveness of the intervention. Our results suggest that using innovative active methodologies seems to be an effective tool on different motivational variables of the students, such as: support for autonomy, support for social relationships, autonomy, intrinsic motivation, identified motivation and external motivation. Despite this, more studies are needed to determine significantly the influence of gamification on motivation in PE classes.

Keywords: gamification, corporal expression, physical education, motivation, dance.

\section{Introducción}

En la actualidad, la falta de motivación y compromiso del alumnado durante las clases de Educación Física (EF) son uno de los mayores retos para los docentes en Educación Secundaria Obligatoria (ESO). Este problema se acentúa cuando se disponen a trabajar actividades

Fecha recepción: 18-11-20. Fecha de aceptación: 30-04-21

Mateo Real-Pérez

mateo.realperez@gmail.com relacionadas con la expresión corporal, contenidos que con frecuencia los docentes son reticentes a incluir en su programación, influyendo así también posiblemente en la motivación del alumnado hacia los mismos (Arias, Fernández, \& Valdés, 2021; Matanim \& Collier, 2003; Ruiz, Graupera, Moreno, \& Rico, 2010; Villard, 2014) Diversos estudios han demostrado que, para hacer frente a los posibles problemas motivacionales que presenta la enseñanza de este contenido en Educación Física, es necesario ofrecer recursos metodológicos y propuestas innovadoras que generen experiencias positivas en el 
alumnado (Sevil, Aibar,Abós, Julián, \& García-González, 2015).

La regulación de la motivación en las clases de EF resulta determinante para fomentar un estilo de vida saludable (Sánchez-Oliva, Sánchez-Miguel, Leo, Kinnafick, \& García-Calvo, 2014). Por ello, en las últimas décadas el estudio de la motivación se determina como un factor importante a tener en cuenta en esta asignatura, siendo la Teoría de la Autodeterminación (TAD) (Deci \& Ryan, 2000) uno de los marcos teóricos más detallados. Según esta teoría, el grado más alto de motivación autodeterminada es la motivación intrínseca, que es aquella que explica la participación voluntaria en una determinada actividad debido al interés, la satisfacción y el placer que se obtiene en la propia realización de la misma (Deci \& Ryan, 2000; Sanchez-Oliva et al., 2014).

Dentro de las metodologías docentes, una de las propuestas más innovadoras para motivar y favorecer el aprendizaje del alumnado es la gamificación. Esta metodología se basa en la aplicación de mecánicas propias del juego en un contexto que, a priori, no debería ser lúdico, con la finalidad de utilizar diferentes elementos relativos al diseño, la estética o las estrategias para involucrar al alumnado. De este modo, se pretende favorecer la motivación, fomentar la participación activa, promover el aprendizaje y resolver problemas (Bozkurt \& Durak, 2018; Lee \& Hammer, 2015; Pérez-López, 2016; Zichermann \& Cunningham, 2011). Con este tipo de experiencias, se busca mantener a los jugadores/ alumnos motivados hacia la participación en el juego (Bozkurt \& Durak, 2018; Pérez-López, 2016).

Algunos referentes en Educación Secundaria valoran de forma positiva el uso de la gamificación como estrategia motivacional para el aprendizaje de hábitos de vida saludable y la práctica regular de ejercicio físico (Monguillot, González, Zurita, Almirall, \& Guitert, 2015). Además, consideran este tipo de metodología como una herramienta potente para movilizar conocimientos y afianzar el proceso de enseñanza-aprendizaje. En este sentido, si hacemos referencia a los contenidos de expresión corporal, tradicionalmente se han relacionado con pensamientos estereotipados y prejuicios sobre las diferentes actividades llevadas a cabo (Sevil, et al., 2015; Villard, 2014). Por ello, es necesario plantear propuestas innovadoras que atraigan el interés del alumnado, motivándole hacia la realización de este tipo de contenidos.

Por ello, presentamos una estrategia de gamificación en el ámbito educativo, llevada a cabo con alumnos de
ESO denominada «ÁFRICA: La leyenda de Faro», diseñada para trabajar los contenidos correspondientes al bloque de expresión corporal a través de la danza. Para ello, seguiremos las pautas estipuladas según la normativa vigente dentro del Sistema Educativo español y la Consejería de Educación y Deporte de la Junta de Andalucía (CEJA, 2016).

En nuestra propuesta utilizaremos la danza africana como eje vertebrador de nuestra línea de actuación. Consideramos que los valores de este estilo de danza, tanto a nivel social como rítmico, motriz y expresivo, son de una incalculable riqueza (Acogny, 1994), y pensamos que puede contribuir a un acercamiento a otras culturas y formas de vida desde el respeto y la solidaridad, mediante el disfrute de la danza (De Miguel \& Mora, 1999; Zurdo \& Padilla, 2003).

Por tanto, teniendo en cuenta el marco teórico y las investigaciones previas, el objetivo de nuestro estudio fue contrastar el efecto de una intervención en una unidad didáctica (UD) aplicando metodologías activas como la gamificación, en comparación con otra en la que se utilizaron estilos de enseñanza tradicionales, sobre la motivación situacional respecto a los contenidos de expresión corporal, en alumnos de $3^{\circ}$ y $4^{\circ}$ de Educación Secundaria Obligatoria (ESO).

\section{Metodología}

Se llevó a cabo un estudio de intervención en el que se compararon dos metodologías aplicadas a la enseñanza de los contenidos de expresión corporal marcados por la normativa vigente en ESO (CEJA, 2016). Para ello, se contó con un grupo experimental (GE) al que se aplico una experiencia de gamificación con utilización de elementos y dinámicas características de esta propuesta metodológica no convencional; frente a un grupo control (GC), donde se aplicaron técnicas de enseñanza basadas en estilos tradicionales como son la instrucción directa y la asignación de tareas.

\section{Participantes}

En el presente estudio participaron un total de 112 alumnos de una muestra obtenida por conglomerado, distribuidos en cuatro clases de $3^{\circ}$ y $4^{\circ}$ de ESO, pertenecientes a un colegio público, con edades comprendidas entre 15 y 17 años $(M$ edad=15.5; DT=.537). Tras la aplicación de los criterios de inclusión (asistencia al $85 \%$ de las sesiones de la UD (UD) y cumplimentación de los cuestionarios en las dos mediciones), la muestra final estuvo compuesta por 98 alumnos $(M$ edad $=15.47$; 
$\mathrm{DT}=.541), 40$ de género masculino $(M$ edad $=15.53$; $\mathrm{DT}=.506)$ y 58 de género femenino $(M$ edad $=15.43$; $\mathrm{DT}=.565)$, divididos en un grupo control $(\mathrm{GC} ; \mathrm{n}=49)$ y un grupo experimental (GE; $n=49)$. El docente de Educación Física fue el encargado de llevar a cabo las diferentes sesiones durante la UD. Además, éste recibió formación específica para el desarrollo de la misma, así como para optimizar el cumplimiento de las condiciones propuestas tanto en GC como en GE.

\section{Instrumentos}

Apoyo a las Necesidades Psicológicas Básicas (NBP)

Se utilizó una adaptación del Cuestionario de Apoyo a las Necesidades Psicológicas Básicas (CANPB; SánchezOliva, Leo, Amado, Cuevas, \& García-Calvo, 2013) adecuado al contexto de la expresión corporal (SánchezOliva, Marcos, Amado, González, \& García-Calvo, 2012), cuya adaptación consistió en modificar la redacción de la frase introductoria «En Educación Física nuestro profesor/a...» por «En las clases de expresión corporal en Educación Física nuestro profesor/a...». El instrumento consta de 12 ítems agrupados en tres factores. El primer factor se corresponde al apoyo de la competencia (Formado por cuatro ítems, ej.: «Nos anima a que confiemos en nuestra capacidad para hacer bien las tareas»); el segundo factor hace referencia al apoyo de la autonomía (Cuatro ítems, ej.: «Nos pregunta a menudo sobre nuestras preferencias con respecto a las actividades a realizar»); y el tercer factor se refiere al apoyo de las relaciones sociales (Cuatro ítems, ej.: «Fomenta en todo momento las buenas relaciones entre los compañeros/as de clase»). Se midió a través de una escala de Likert de 5 puntos, $(1=$ «Totalmente en desacuerdo», $5=$ «Totalmente de acuerdo»).

\section{Satisfacción de las Necesidades Psicológicas Básicas}

Utilizamos la versión traducida al castellano y adaptada a la Educación Física (Moreno, González-Cutre, Chillón, \& Parra, 2008) de la Escala de Medición de las Necesidades Psicológicas Básicas (BPNES; Vlachopoulos \& Michailidou, 2006). Este instrumento contenía el siguiente encabezado adaptado al contexto del estudio: «En las clases de expresión corporal en Educación Física...», seguido de 12 ítems que evaluaban la satisfacción de las tres necesidades psicológicas agrupados en tres factores (cuatro ítems por factor) para medir la percepción de autonomía (ej.: «Los ejercicios que realizamos en las clases de expresión corporal se ajustan a mis intereses»); la percepción de competencia (ej.:
«Realizo los ejercicios eficazmente en las clases de expresión corporal»); y la percepción de relación con los demás (ej.: «Siento que me puedo comunicar abiertamente con mis compañeros en las clases de expresión corporal»). Se midió a través de una escala de Likert de 5 puntos, $(1=$ «Totalmente en desacuerdo», $5=$ «Totalmente de acuerdo»).

\section{Motivación}

Se utilizó el Cuestionario de Motivación en la Educación Física (CMEF; Sánchez-Oliva, et al., 2012). Este cuestionario cuenta como frase inicial «Yo participo en las clases de expresión corporal en Educación Física...», seguida de 20 ítems (cuatro por factor) agrupados en cinco factores, para medir valores relacionados con la motivación intrínseca (ej.: «Porque la expresión corporal es divertida»), la regulación identificada (ej.: «Porque la expresión corporal me aporta conocimientos y habilidades que considero importantes); la regulación introyectada (ej.: «Porque lo veo necesario para sentirme bien conmigo mismo») la regulación externa (ej.: «Para demostrar al profesor/a y compañeros/as mi interés por la expresión corporal») y la desmotivación (ej.: «Pero realmente siento que estoy perdiendo mi tiempo con la expresión corporal»). Se midió a través de una escala de Likert de 5 puntos, $(1=$ «Totalmente en desacuerdo», $5=$ «Totalmente de acuerdo»).

\section{Predisposición hacia la Educación Física}

Utilizamos la Escala de Predisposición hacia la EF (PEPS; Hilland, Stratton, Vinson, \& Fairclough, 2009) adaptada y validada al contexto español (Granero-Gallegos \& Baena-Extremera, 2016). Este instrumento fue adaptado modificando la frase inicial al contenido de la UD «En la unidad de expresión corporal...», seguido de 11 ítems para medir la predisposición motivacional hacia la EF en dos dimensiones: percepción de esfuerzo en EF (seis ítems; ej: «Tengo la suficiente confianza para participar en las clases de expresión corporal») y percepción de habilidad en EF (cinco ítems; ej: «Me siento muy capaz en expresión corporal»). El esfuerzo se mide a partir de la media de los seis reactivos que representan la evaluación de ítems correspondientes a la participación en las clases de EF y que reflejan la actitud afectiva y cognitiva (Welk, 1999). La habilidad se mide a partir de la media de los otros cinco ítems indicativos de la percepción de competencia y auto-eficacia en EF (Granero-Gallegos \& Baena-Extremera, 2016). Se midió a través de una escala de Likert de 5 puntos, $(1=$ «Totalmente en desacuerdo», $5=$ «Totalmente de acuerdo»). 


\section{Diversión/ aburrimiento}

Se utilizó el Sport Satisfaction Instrument (SSI; Nicholls \& Duda, 1992) adaptado al español y validado en las clases de EF (SSI-EF; Baena-Extremera, Granero-Gallegos, Bracho, \& Pérez-Quero, 2012). Esta escala está compuesta por 8 ítems agrupados en dos factores: cinco ítems medían la satisfacción/diversión (ej.: «Normalmente encuentro las clases de expresión corporal interesantes») y tres ítems hacían referencia al aburrimiento (ej.: «En las clases de expresión corporal, deseo que las clases terminen rápidamente». Se midió a través de una escala de Likert de 5 puntos, $(1=$ «Totalmente en desacuerdo», $5=$ «Totalmente de acuerdo»).

\section{Procedimientos}

\section{Recogida de datos}

Para la recogida de los datos contactamos con el centro de enseñanza con la finalidad de informarle de los objetivos del estudio y pedirle su consentimiento en la administración de diferentes cuestionarios. Tras la aprobación del equipo directivo y del departamento de EF se pidió consentimiento informado a los padres o tutores de los alumnos.

El estudio se realizó a lo largo de una UD de 5 semanas, en la que trabajamos los contenidos propios del bloque de expresión corporal para $3^{\circ}$ y $4^{\circ}$ de ESO integradas en la programación didáctica anual del profesor de EF del centro, utilizando la Danza Africana como eje vertebrador de las clases. Las clases tuvieron una duración de 60 minutos y una frecuencia de 2 sesiones semanales, completando un total de seis sesiones.

La toma de datos se realizó antes de comenzar y una vez finalizada la UD. Se utilizó el aula de clase durante la hora de tutoría, con presencia del investigador principal y en ausencia del docente de EF.

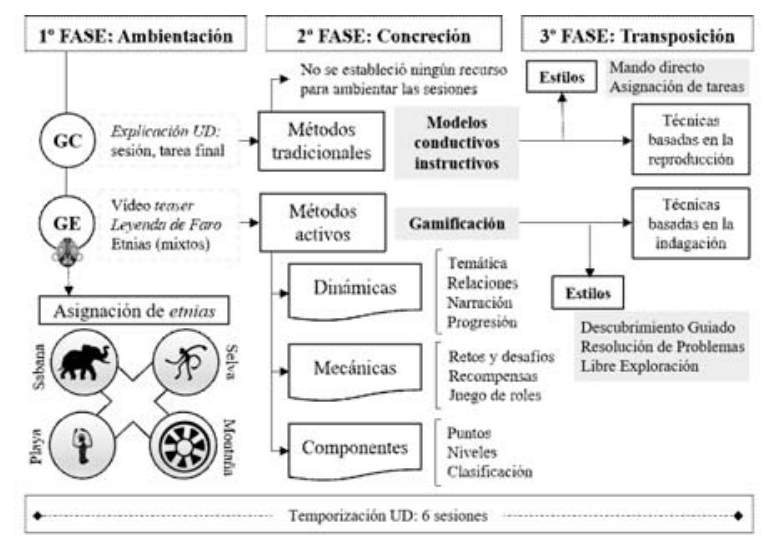

Figura 1. Desarrollo de la intervención docente.

\section{Desarrollo de la intervención docente}

La UD se dividió en seis pantallas didácticas, en las cuales el alumnado trabajó diferentes actividades relacionadas con el bloque de contenidos de expresión corporal, utilizando la danza africana como elemento principal de la temática del proyecto. A continuación, mostramos la estructura de nuestra intervención docente (Figura 1):

\section{Análisis estadístico}

Se llevó a cabo un análisis de frecuencias para las variables de género, edad, curso y grupo de estudio. Para ver la influencia de la variable género sobre la motivación situacional respecto al contenido de expresión corporal, se realizó un análisis comparativo de las medias (ANOVA), en el que se tuvieron en cuenta los valores medios y la desviación típica del factor género respecto a las variables de apoyo a las NPB, satisfacción de las NPB y los diferentes tipos de motivación en las medidas obtenidas en el pre-test. Para cuantificar los cambios producidos durante el período de intervención, se compararon los datos obtenidos en la medición pretest y post-test a través de cuestionarios. Para ello, se tuvo en cuenta las medias marginales estimadas intrasujeto (Grupo*Medida) y la desviación típica para cuantificar la interacción entre las variables y su evolución longitudinal a través de un ANOVA de medidas repetidas, aplicando la prueba post hoc por Bonferroni. El análisis de todas las variables del estudio se llevó a cabo usando el paquete estadístico SPSS versión 25 (SPSS,inc, IBM).

\section{Resultados}

En la tabla 1 se muestra el análisis de frecuencias con indicación de la $n$ por sexo, curso, edad y grupos (GC-GE).

Por otro lado, en la tabla 2 se muestra la comparativa intra-sujetos de los efectos de una metodología basada en la gamificación sobre la motivación situacional respecto al contenido de expresión corporal en el grupo experimental comparado con el uso de metodologías tradicionales basadas en la instrucción directa en el grupo control. Para facilitar su interpretación, los datos presentados en dicha tabla se ven plasmados en las figuras 2 y 3, donde se puede observar cómo, a pesar de no mostrar diferencias significativas estadísticamente, la tendencia del grupo experimental muestra valores de mejora más altos respecto al grupo control en los ítems que hacen referencia al apoyo a la autonomía, apoyo a las 
Tabla 1.

\begin{tabular}{lccc} 
Análisis de frecuencias & & & \\
\hline \multirow{2}{*}{ Género } & & Frecuencia & Porcentaje (\%) \\
\hline \multirow{2}{*}{ Curso } & Masculino & 40 & 40,8 \\
& Femenino & 58 & 59,2 \\
\hline \multirow{2}{*}{ Edad } & $3^{\circ}$ & 54 & 55,2 \\
& $4^{\circ}$ & 44 & 44,8 \\
\hline \multirow{6}{*}{ Grupo } & 15 & 54 & 55,1 \\
& 16 & 42 & 42,9 \\
& 17 & 2 & 2 \\
& Control & 49 & 50 \\
& Masculino & 19 & 38,78 \\
& Femenino & 30 & 61,22 \\
& Experimental & 49 & 50 \\
& Masculino & 21 & 42,86 \\
& Femenino & 28 & 57,14 \\
\hline
\end{tabular}

Tabla 2

Análisis intra-sujeto de la motivación situacional en función del grupo.

\begin{tabular}{|c|c|c|c|c|c|c|c|}
\hline & \multicolumn{2}{|c|}{ Grupo Control } & \multicolumn{2}{|c|}{ Grupo Experimental } & \multicolumn{3}{|c|}{$\begin{array}{l}\text { Efecto Interacción } \\
\text { Medida*Grupo }\end{array}$} \\
\hline & Pre-Test & Post-Test & Pre-Test & Post-Test & $M C$ & $F$ & $p$ \\
\hline Apoyo Autonomía & $3.68(.13)$ & $4.02(.12)$ & $2.98(.13)$ & $3.66(.12)$ & 1.418 & 2.134 & .147 \\
\hline Apoyo Competencia & $3.89(.11)$ & $4.18(.11)$ & $3.86(.11)$ & $4.06(.11)$ & .085 & .164 & .687 \\
\hline Apoyo Relaciones & $4.30(.13)$ & $4.46(.11)$ & $3.86(.13)$ & $4.19(.11)$ & .32 & .79 & .376 \\
\hline Autonomía & $3.30(.12)$ & $3.50(.12)$ & $2.76(.12)$ & $3.18(.12)$ & .571 & 1.17 & .282 \\
\hline Competencia & $3.66(.11)$ & $3.79(.10)$ & $3.73(.11)$ & $3.81(.10)$ & .044 & .149 & .701 \\
\hline Relaciones & $4.02(.13)$ & $4.11(.12)$ & $3.93(.13)$ & $3.91(.12)$ & .154 & .53 & .468 \\
\hline & $3.70(.13)$ & $3.92($. & $2.93(.13)$ & 3.21( & .051 & .087 & .768 \\
\hline Identificada & $3.47(.13)$ & $3.61(.13)$ & $2.68(.13)$ & $2.95(.13)$ & 205 & .485 & 488 \\
\hline Introyectada & $3.46(.12)$ & $3.52(.11)$ & $2.92(.12)$ & $2.84(.11)$ & 195 & .474 & .493 \\
\hline Externa & $3.24(.11)$ & $3.46(.12)$ & $2.91(.11)$ & $3.27(.12)$ & 276 & .505 & 479 \\
\hline Desmotivación & $2.57(.14)$ & $3.10(.15)$ & $2.53(.14)$ & $2.79(.15)$ & .907 & 1.180 & .280 \\
\hline
\end{tabular}

relaciones sociales, satisfacción de la autonomía, motivación intrínseca, motivación identificada y motivación externa; así como una disminución de la desmotivación. En cuanto al grupo control, muestra una tendencia mayor en cuanto al apoyo a la competencia, satisfacción de la competencia, relaciones sociales y motivación introyectada; además de aumentar el nivel de desmotivación tras el período de intervención.
Respecto a los datos obtenidos en función de la predisposición hacia los contenidos de expresión corporal, en la figura 4 se observa una tendencia positiva en los resultados en cuanto a habilidad y diversión en el grupo experimental, mientras que en el grupo control se muestra una tendencia creciente en los ítems relacionados con el esfuerzo y el aburrimiento. Sin embargo, tal y como se puede observar en la tabla 3 estos resultados no son estadísticamente significativos.
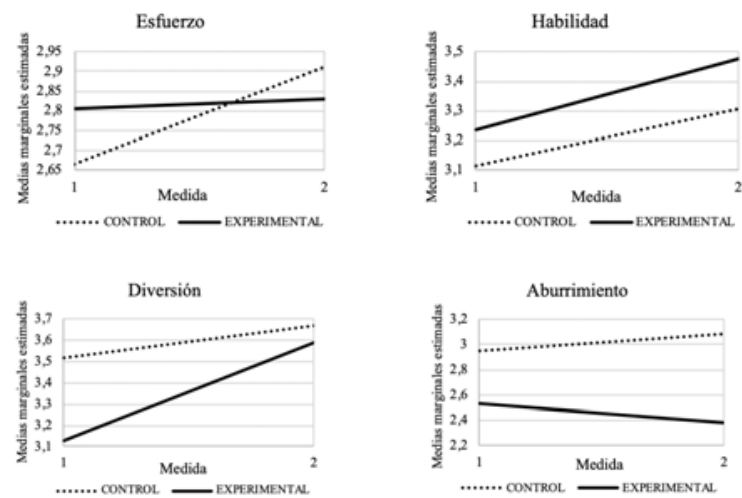

Figura 4. Análisis gráfico de los resultados según la percepción de le expresión corporal.

Tabla 3

Análisis intra-sujeto de la predisposición hacia la $E C$ en función del grupo.

\begin{tabular}{lccccccc}
\hline & \multicolumn{2}{c}{ Grupo Control } & \multicolumn{2}{c}{ Grupo Experimental } & \multicolumn{3}{c}{$\begin{array}{c}\text { Efecto Interacción } \\
\text { Medida*Grupo }\end{array}$} \\
\cline { 2 - 8 } & Pre-Test & Post-Test & Pre-Test & Post-Test & MC & $F$ & $p$ \\
\hline Esfuerzo & $2.66(.08)$ & $2.91(.11)$ & $2.80(.08)$ & $2.83(.11)$ & .597 & 1.525 & .220 \\
Habilidad & $3.11(.11)$ & $3.31(.13)$ & $3.24(.11)$ & $3.48(.13)$ & .024 & .056 & .814 \\
Diversión & $3.52(.14)$ & $3.67(.11)$ & $3.13(.14)$ & $3.59(.11)$ & 1.187 & 1.814 & .181 \\
Aburrimiento & $2.95(.16)$ & $3.09(.15)$ & $2.52(.16)$ & $2.38(.15)$ & .993 & 1.038 & .311 \\
\hline
\end{tabular}
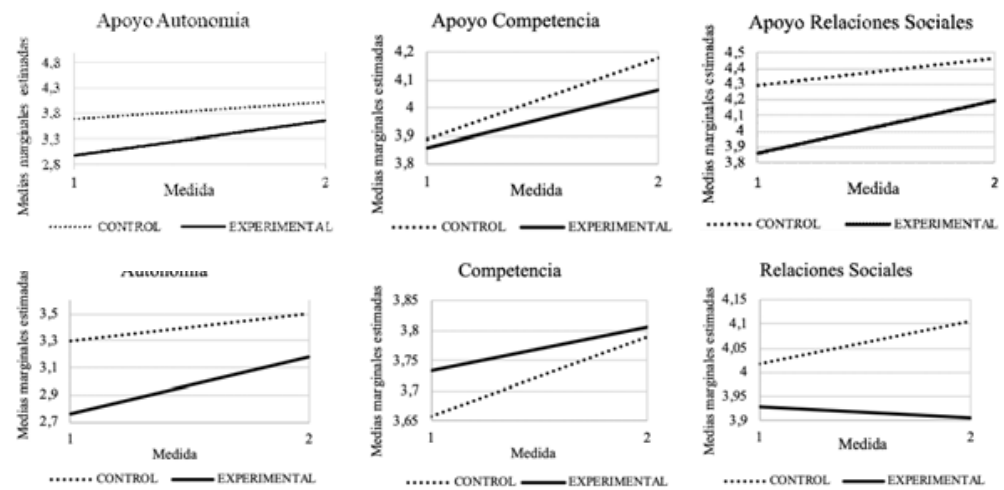

........ CONTROL — EXPERIMENTAL

Figura 2. Análisis de los resultados según el apoyo y la satisfacción de las NPB.
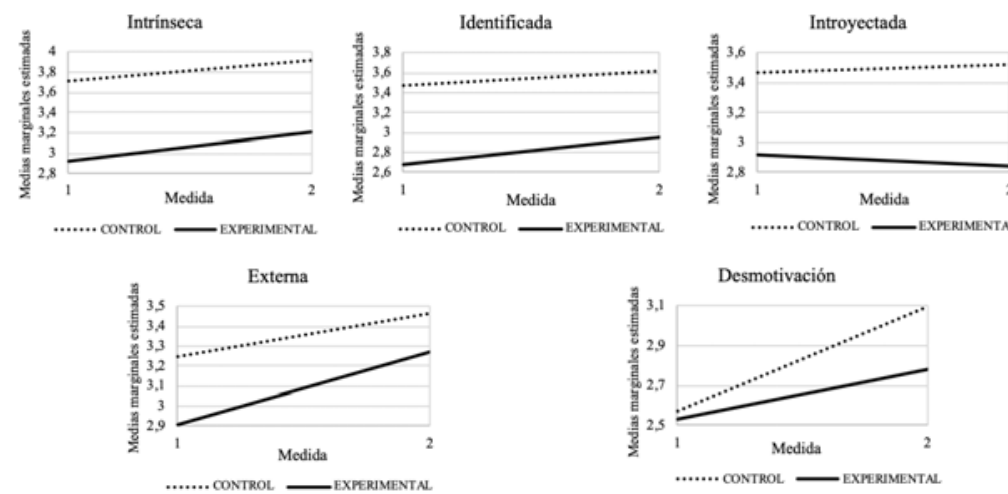

Figura 3. Análisis de los resultados según el tipo de motivación.

\section{Discusión}

El propósito de este estudio fue analizar, teniendo en cuenta las postulaciones de la TAD (Deci \& Ryan, 2000), la motivación situacional respecto al contenido de expresión corporal en función de la metodología de enseñanza. Tras analizar los resultados del estudio, podemos sugerir: (1) el GE muestra valores de mejora más altos respecto al GC en los ítems que hacen referencia al apoyo a la autonomía, apoyo a las relaciones sociales, satisfacción de la autonomía, motivación intrínseca, motivación identificada y motivación externa; así como una disminución de la desmotivación; (2) tendencia positiva en la percepción de habilidad y diversión en el GE, mientras que en el GC aumenta los parámetros de esfuerzo y aburrimiento. Estos resultados sugieren la importancia que puede tener el uso de metodologías activas como la 
gamificación sobre la motivación situacional respecto a los contenidos de expresión corporal en alumnos de ESO.

En primer lugar, si analizamos la comparativa intrasujeto de los efectos de una metodología basada en la gamificación sobre la motivación situacional después de haber realizado la UD en el período de intervención, podemos observar ciertas tendencias positivas en el GE en diferentes dimensiones de la TAD (Deci \& Ryan, 2000) como son: apoyo a la autonomía, apoyo a las relaciones sociales, satisfacción de la autonomía, motivación intrínseca, motivación identificada y motivación externa. Además de mostrar índices más elevados de percepción de habilidad y diversión en las clases. En cuanto al GC, encontramos una tendencia mayor en cuanto a las medias marginales estimadas de: apoyo a la competencia, satisfacción de la competencia, satisfacción de las relaciones sociales, motivación introyectada y desmotivación. Además de una mayor percepción del esfuerzo y el aburrimiento después del período de intervención.

Si tenemos en cuenta estos datos, el hecho de que el GE manifieste menores medias tanto en el apoyo como en la satisfacción de la competencia, puede deberse fundamentalmente a que durante las clases de la UD del GC, las técnicas de enseñanza basadas en la instrucción directa favorecen que los alumnos imiten un modelo determinado, lo que facilita que tengan la sensación de ser competentes al ser capaces de reproducir secuencias prestablecidas; mientras que los alumnos del GE se enfrentan a una mayor incertidumbre al tener que indagar por descubrimiento guiado en cómo realizar los diferentes ejercicios propuestos de forma competente en base a los objetivos propuestos (Zapatero, 2017).

En cuanto a la satisfacción de las relaciones sociales, mientras que el GE se agrupa en grupos heterogéneos (etnias) conformados por los mismos sujetos durante toda la UD; dentro del GC existían diferentes modos de agrupamiento, favoreciéndose de este modo que los alumnos interactúen libremente con los distintos compañeros de clase en función de la actividad. Esta diferencia podría explicar la mejora de la satisfacción en cuanto a relaciones sociales en el GC respecto al GE; aunque, por otro lado, durante el transcurso de las diferentes sesiones el alumnado del GE vio reforzados los lazos entre los componentes de las diferentes etnias (grupos heterogéneos), aprendiendo a colaborar para conseguir ciertos objetivos comunes. Desde el punto de vista metodológico, sería necesario plantear nuevas dinámicas que afiancen los parámetros relacionados con la satisfacción de las relaciones sociales entre los diferentes componentes de cada etnia.

Continuando con el análisis de los ítems que hacen referencia a la motivación según la TAD (Deci \& Ryan, 2000), cabe destacar que tras el período de intervención el GE muestra un aumento de la motivación intrínseca (aquella que incita a hacer actividades de expresión corporal por el mero disfrute), identificada (relacionada con la comprensión y puesta en valor de la expresión corporal y sus beneficios) y externa (posiblemente influida por las dinámicas propias de la gamificación como son los sistemas de recompensa o la tabla de puntuaciones); en el GC vemos un aumento de la motivación introyectada (cuando se realizan las clases de expresión corporal para evitar los sentimientos de culpa) y la desmotivación. De este modo, los resultados sugieren que una UD basada en una experiencia de gamificación podría mejorar la motivación situacional del grupo de clase respecto a los contenidos de expresión corporal, lo que favorecería que el alumnado tenga mayor predisposición a la participación en las sesiones de Educación Física.

Finalmente, cuando analizamos de forma comparativa las variables dependientes intra-sujeto no obtuvimos diferencias estadísticamente significativas $(\mathrm{p}<0.5)$. A pesar de haber utilizado instrumentos válidos $\mathrm{y}$ fiables usados también en otros estudios sobre motivación basados en la TAD (Sevil, et al., 2015; Almolda-Tomás, Sevil-Serrano, Julián-Clemente, Abarca-Sos, Aibar-Solana, \& García-González, 2014; Borg, 1982; GraneroGallegos, Baena Extremera, Sánchez-Fuentes \& Martínez-Molina, 2014; Sanchez-Oliva, et al., 2014; Sevil, et al., 2015; Shen, et al., 2016), Esto puede deberse a diversos factores, como pueden ser la baja potencia muestral del estudio con una $n$ pequeña en comparación con otros estudios publicados (Sevil, et al., 2015; Sanchez-Oliva, et al., 2014). También puede influir el corto período de tiempo de la UD, ya que trabajamos los contenidos de expresión corporal durante seis sesiones en un período de cinco semanas. Mientras otros estudios comparativos en función del género (Sevil, et al., 2015) utilizaron un período de intervención de 10 sesiones en 5 semanas mostrando valores significativos.

Aunque los resultados estadísticos comparativos de las diferentes variables dependientes relacionadas con la motivación situacional respecto al contenido de expresión corporal no son significativos en el análisis intrasujeto, nos gustaría destacar que, si hacemos referencia a las figuras que describen los resultados, podemos observar una tendencia positiva en cuanto al apoyo a la 
autonomía, apoyo a las relaciones sociales, satisfacción de la autonomía, motivación intrínseca, motivación identificada y motivación externa; así como una disminución de la desmotivación en el GE que ha sido tratado con metodologías basadas en una experiencia de gamificación.

\section{Fortalezas y limitaciones}

Como fortaleza señalar que hasta la fecha, este es el primer estudio que hace referencia a la cuantificación con datos estadísticos aplicando como intervención contenidos de Danza Africana. Aunque serían necesarias futuras investigaciones que sirvan para cuantificar los efectos de diferentes propuestas metodológicas activas innovadoras que permitan intervenciones docentes más efectivas. Por otro lado, con una duración mayor del período de intervención (en el que hemos querido respetar la duración estándar de una UD), podrían corroborarse dichos resultados respecto a la motivación situacional y la predisposición del alumnado, siendo una de las limitaciones de nuestro estudio. Otra limitación de nuestro estudio es que no mostramos resultados en función del sexo (data non show), ya que hemos pretendido analizar nuestra intervención con una muestra standard de la realidad educativa de las aulas de la ESO. No obstante, en la tabla 1 de descriptivos se observa la homogeneidad en la distribución de los GC y GE en cuanto al sexo.

\section{Conclusiones}

Los resultados indican que una intervención docente basada en estrategias y metodologías activas innovadoras de enseñanza a través de una experiencia de gamificación, podría aumentar la percepción sobre la diversión y el clima motivacional, mejorando la predisposición de los discentes respecto a los contenidos de expresión corporal. Sin embargo, es necesario un mayor número de estudios para confirmar de forma significativa nuestros resultados.

\section{Agradecimientos}

Este trabajo ha sido subvencionado por la Asociación Universitaria de Danza UCAdanza de la Universidad de Cádiz. Además, nos gustaría dar las gracias al alumnado participante en este estudio.

\section{Referencias}

Acogny, G. (1994). Danse Africaine (Weigarten). Berlin, Germany: Weigarten.

Almolda-Tomás, F. J., Sevil-Serrano, J., Julián-Clemente, J. A., Abarca-Sos, A., Aibar-Solana, A., \& GarcíaGonzález, L. (2014). Application of teaching strategies for improving students' situational motivation in physical education. Electronic Journal of Research in Educational Psychology, 12(2), 391-418. https://doi.org/10.14204/ejrep.33.13148

Arias, J. R., Fernández, B., \& Valdés, R. (2021). Actitudes hacia la Expresión Corporal en el ámbito de la asignatura de Educación Física: Un estudio con alumnado de Educación Secundaria Obligatoria (Attitudes towards Body Expression in the field of Physical Education subject: A study with Compulsory Se. Retos, 2041(41), 596-608. https://doi.org/ $10.47197 /$ retos.v0i41.83296

Baena-Extremera, A., Granero-Gallegos, A., Bracho, C. A., \& Pérez-Quero, F. J. (2012). Versión española del «Sport Satisfaction Instrument (SSI)» adaptado a la Educación Física. Revista de Psicodidactica, 17(2), 377-395. https://doi.org/10.1387/ Rev.Psicodidact.4037

Borg, G. (1982). Psychophysical bases of perceived exertion. Medicine and Science in Sports and Exercise, 14(5), 377-381. https://doi.org/10.1249/ 00005768-198205000-00012

Bozkurt, A., \& Durak, G. (2018). A Systematic Review of Gamification Research. International Journal of Game-Based Learning, 8(3), 15-33. https: / / doi.org/ 10.4018/ijgbl.2018070102

CEJA. Orden de 14 de julio de 2016, por la que se desarrolla el currículo correspondiente a la Educación Secundaria Obligatoria en la Comunidad Autónoma de Andalucía y se regulan determinados aspectos de la atención a la diversidad (2016).

De Miguel, I., \& Mora Verdeny, J. M. (1999). La danza africana , una historia sin palabras Posibles adaptaciones en el área de educación física. Aula de Innovación Educativa, 82, 13-18.

Deci, E. L., \& Ryan, R. M. (2000). The «What» and «Why» of Goal Pursuits: Human Needs and the SelfDetermination of Behavior. Psychological Inquiry, 11(4), 227-268. https://doi.org/10.1207/ S15327965PLI1104_01

Granero-Gallegos, A., Baena Extremera, A., SánchezFuentes, J. A., \& Martínez-Molina, M. (2014). Per- 
files motivacionales de apoyo a la autonomía, autodeterminación, satisfacción, importancia de la educación física e intención de práctica física en tiempo libre. Cuadernos de Psciología Del Deporte, 14(2), 59 69.

Granero-Gallegos, Antonio, \& Baena-Extremera, A. (2016). Validación española de la Escala de Predisposición hacia la Educación Física (PEPS) en educación secundaria. Retos: Nuevas Tendencias En Educación Física, Deporte y Recreación, 30, 125-131.

Hilland, T. A., Stratton, G., Vinson, D., \& Fairclough, S. (2009). The physical education predisposition scale: Preliminary development and validation. Journal of Sports Sciences, 27(14), 1555-1563. https: / / doi.org/ $10.1080 / 02640410903147513$

Lee, J., \& Hammer, J. (2015). Gamification in Education/ : What , How , Why Bother/ ? Gamification in Education/: What, How , Why Bother/ ? Academic Exchange Quarterly, 15(2), 146-151.

Matanim, M. , \& Collier, C. (2003). Longitudinal analysis of preservice teachers' beliefs about teaching physical education. Journal of Teaching in Physical Education, 22(2), 153-168.

Miller, K.A., Deci, E. L., \& Ryan, R. M. (1988). Intrinsic Motivation and Self - Determination in Human Behavior. Contemporary Sociology, 17(2), 253. https: / /doi.org/10.2307/2070638

Monguillot, M., González, C., Zurita, C., Almirall, L., \& Guitert, M. (2015). Play the Game: gamificación y hábitos saludables en educación física. Apunts: Educació Física i Esports, (119), 71-79.

Moreno, J.A., González-Cutre, D., Chillón, M. y Parra, N. (2008). Adaptación a la educación física de la escala de las necesidades psicológicas básicas en el ejercicio (BPNES). Revista Mexicana de Psicología, 25(2006), 295-303.

Nicholls, J. G., \& Duda, J. L. (1992). Dimensions of Achievement Motivation in Schoolwork and Sport. Journal of Educational Psychology, 84(3), 290-299.

Pérez-López, I. J. (2016). No te la juegues con tu salud, gamíficala: «La amenaza de los Sedentaris.» Habilidad Motriz, 46, 42-48.

Ruiz, L. M., Graupera, J. L., Moreno, J. A., \& Rico, I. (2010). Social Preferences for Learning among Adolescents in Secondary Physical Education. Journal of Teaching in Physical Education, 29(1), 3-20. https: / /doi.org/10.1123/jtpe.29.1.3

Sánchez-Oliva, D., Leo, F. M. L., Amado, D., Cuevas, R., \& García-Calvo, T. (2013). Desarrollo y validación del cuestionario de apoyo a las necesidades psicológicas básicas en educación física. European Journal of Human Movement, 30(May 2014), 53-71.

Sanchez-Oliva, D., Sanchez-Miguel, P. A., Leo, F. M., Kinnafick, F.-E., \& García-Calvo, T. (2014). Physical Education Lessons and Physical Activity Intentions Within Spanish Secondary Schools: A SelfDetermination Perspective. Journal of Teaching in Physical Education, 33(2), 232-249. https: / / doi.org/ 10.1123/jtpe.2013-0043

Sánchez-Oliva, David, Marcos, F. M. L., Amado, D., González, I., \& García-Calvo, T. (2012). Desarrollo de un cuestionario para valorar la motivación en educación física. Revista Iberoamericana de Psicología Del Ejercicio y El Deporte, 7(2), 227-250.

Sevil, J., Aibar, A., Abós, Á., Julián, J. A., \& GarcíaGonzález, L. (2015). Gender and corporal expression activity in physical education. European Physical Education Review, 22(3), 372-389. https: / / doi.org/ 10.1177/1356336x15613463

Shen, B., Chen, A., Tolley, H., \& Scrabis, K. A. (2016). Gender and Interest-Based Motivation in Learning Dance. Journal of Teaching in Physical Education, 22(4), 396-409. https://doi.org/10.1123/jtpe.22.4.396

Villard, M. (2014). Percepciones del profesorado de Educación Física de Educación Secundaria sobre el papel de la expresión corporal en el currículum en Andalucía. Universidad de Huelva, España.

Vlachopoulos, S. P., \& Michailidou, S. (2006). Development and initial validation of a measure of autonomy, competence, and relatedness in exercise: The Basic Psychological Needs in Exercise Scale. Measurement in Physical Education and Exercise Science, 10(3), 179-201. https://doi.org/10.1207/ s15327841mpee1003_4

Welk, G. J. (1999).TheYouth Physical Activity Promotion Model: A Conceptual Bridge Between Theory and Practice. Quest, 51(1), 5-23. https://doi.org/ 10.1080/00336297.1999.10484297

Zapatero, J. A. (2017). Beneficios de los estilos de enseñanza y las metodologías centradas en el alumno en Educación Física. E-Balonmano. Com: Revista de Ciencias Del Deporte, 13(3), 237-250.

Zichermann, G., \& Cunningham, C. (2011). Gamification By Design - Implementing Game Mechanics in Web and Mobile Apps. O’Reilly Media, Inc. Sebastopol, Canadá. https: / /doi.org/10.1017/CBO9781107415324.004

Zurdo Giménez, R., \& Padilla Moledo, C. (2003). Conocimiento de otras culturas con el cuerpo como vehículo: Danzas étnicas. Expresión, Creatividad y Movimiento. I Congreso Internacional de Expresión Corporal y Educación. Universidad de Salamanca. Zamora, 579-587. 